\title{
Auxiliary branch method and modified nodal voltage equations
}

\author{
A. Reibiger \\ Dresden University of Technology, Department of Electrical Engineering, Mommsenstr. 13, 01062 Dresden, Germany
}

\begin{abstract}
A theorem is presented describing a transformation by means of which it is possible to assign to an elementary multiport with fairly general constitutive equations (including all kinds of controlled sources, nullors, ideal transformers, etc.) a modified multiport with the same all-pole terminal behavior. The branch set of this modified multiport is augmented with so called auxiliary branches whereas its constitutive equations are always in conductance form. Therefore an interconnection of a family of multiports transformed in this manner can always be analyzed by means of a system of nodal voltage equations. It will be shown that this system of equations is equivalent to a system of modified nodal voltage equations set up for the network that is an interconnection of the elementary multiports originally given.
\end{abstract}

\section{Introduction}

For an arbitrary elementary multipole with semi-implicite constitutive equations we consider an algorithm for the construction of a multipole having the same terminal behavior as the given elementary multipole but with constitutive equations in conductance form.

The idea for the construction of this algorithm goes back to a conference paper of Reinschke and Schwarz (1977). Former related results go back to papers of Klein (1958) and his collaborators Engelhardt and Heinz (1970); Thielmann (1973); Kremer (1978).

As an application of the theory of terminal behavior of networks developed in Reibiger (1985, 1986, 2003a) we present a reformulation of this algorithm which makes its network theoretic assumptions and consequences more transparent.

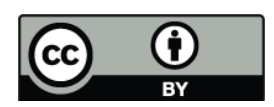

Correspondence to: A. Reibiger (reibiger@iee.et.tu-dresden.de)

\section{Network theoretical background}

In this section we give a short review of results of our research on the foundations of network theory Reibiger (1985) to Reibiger (2003b).

As standing notations we use the symbols $\mathcal{U}, \mathcal{I}$, and $\mathcal{T}$ to denote the space of branch voltage values, the space of branch current values, and the time axis, respectively. We assume that all these spaces are provided with the structure of one-dimensional normed oriented real vector spaces. $\operatorname{Int} \mathcal{T}$ denotes the set of all intervals of the time axis.

The assumptions $\mathcal{U}=\mathbb{R V}, \mathcal{I}=\mathbb{R A}$, and $\mathcal{T}=\mathbb{R}$ s lead to the class of electrical networks and $\mathcal{U}=\mathcal{I}=\mathcal{T}=\mathbb{R}$ to that of normalized networks. In the same manner it is possible to introduce network classes which can be used for modeling mechanical or thermal systems, etc., cf. Koenig et al. (1967); Reinschke and Schwarz (1976).

For each nonvoid finite set $\mathcal{Z}$ and each interval $T \in \operatorname{Int} \mathcal{T}$ we assume that the sets $\mathcal{U}^{\mathcal{Z}},\left(\mathcal{U}^{\mathcal{Z}}\right)^{T}, \mathcal{U}^{\mathcal{Z}} \times \mathcal{I}^{\mathcal{Z}}, \ldots$, $\left(\mathcal{U}^{\mathcal{Z}}\right)^{T} \times\left(\mathcal{I}^{\mathcal{Z}}\right)^{T}$ are provided with the structure of normed linear spaces induced by the structures of the spaces $\mathcal{U}$ and $\mathcal{I}^{1}$. The set $\mathcal{S}:=\bigcup_{T \in \operatorname{Int} \mathcal{T}}\left(\mathcal{U}^{\mathcal{Z}}\right)^{T} \times\left(\mathcal{I}^{\mathcal{Z}}\right)^{T}$ is denoted as the universal signal set on $\mathcal{Z}$.

Let $\mathcal{W} \subseteq \mathcal{S}$, where $\mathcal{S}$ is the universal signal set on $\mathcal{Z}$. Let $\operatorname{sd} \mathcal{W}:=\left\{T \mid \exists_{(u, i) \in \mathcal{W}} T=\operatorname{dom} u\right\}$, and let $\dot{\vee}$ denote the exclusive or. The set $\mathcal{W}$ is denoted as restriction compatible if the following conditions are fulfilled:

(i) $\bigcup_{T^{\prime} \in \operatorname{sd} \mathcal{W}} T^{\prime} \in \operatorname{sd} \mathcal{W}$,

(ii) $|\operatorname{sd} \mathcal{W}|=1 \dot{\vee}\left(\forall_{T, T^{\prime} \in \operatorname{Int}} \mathcal{T} T \in \operatorname{sd} \mathcal{W} \wedge T^{\prime} \subseteq T\right.$ $\left.\Rightarrow T^{\prime} \in \operatorname{sd} \mathcal{W}\right)$

(iii) $\forall_{(u, i) \in \mathcal{W}} \forall_{T \in \operatorname{sd} \mathcal{W}} T \subseteq \operatorname{dom} u \Rightarrow(u|T, i| T) \in \mathcal{W}$.

The set $\mathcal{W} \subseteq \mathcal{S}$ is called properly restriction compatible if $|\operatorname{sd} \mathcal{W}|>1$ otherwise $\mathcal{W}$ is referred to as trivially restriction compatible.

\footnotetext{
${ }^{1}$ As usual in set theory we denote for any two sets $X$ and $Y$ the set of all mappings from $X$ to $Y$ by $Y^{X}$. For each $f \in X$ and $X^{\prime} \subset X$ we denote with $f \mid X^{\prime}$ the restriction of $f$ to $X^{\prime}$.
} 
For each $(u, i) \in \mathcal{W}, b \in \mathcal{Z}$, and $\mathcal{Z}^{\prime} \subset \mathcal{Z},\left(\mathcal{Z}^{\prime} \neq \emptyset\right)$ we denote with $u_{b}, i_{b}$, and $u_{\mathcal{Z}^{\prime}}, i_{\mathcal{Z}^{\prime}}$ the time functions defined for all $t \in \operatorname{dom} u=\operatorname{dom} i$ by $u_{b}(t):=u(t)(b)$ and $i_{b}(t):=i(t)(b)$, or $u_{\mathcal{Z}^{\prime}}(t):=u(t) \mid \mathcal{Z}^{\prime}$, and $i_{\mathcal{Z}^{\prime}}(t):=i(t) \mid \mathcal{Z}^{\prime}$, respectively. Under the same assumptions the set $\mathcal{W}_{\mathcal{Z}^{\prime}}:=\left\{\left(u_{\mathcal{Z}^{\prime}}, i_{\mathcal{Z}^{\prime}}\right) \mid(u, i) \in \mathcal{W}\right\}$ is denoted as the projection of $\mathcal{W}$ generated by $\mathcal{Z}^{\prime}$.

An oriented graph is defined as an ordered triple $(\mathcal{Z}, \mathcal{K}, \mathcal{A})$ of two finite disjoint sets $\mathcal{Z}$ and $\mathcal{K}$ with $(\mathcal{K}=\emptyset \Rightarrow \mathcal{Z}=\emptyset)$ and a map $\mathcal{A}: \mathcal{Z} \rightarrow \mathcal{K} \times \mathcal{K} . \mathcal{Z}$ is the branch set, $\mathcal{K}$ the node set, and $\mathcal{A}$ the incidence mapping of this graph. The incidence map assigns to each branch $b$ the ordered pair $(v, w):=\mathcal{A}(b)$ of its initial and terminal node. Each branch is oriented from its initial to its terminal node.

Let $\mathcal{G}=(\mathcal{Z}, \mathcal{K}, \mathcal{A})$ and $\overline{\mathcal{G}}=(\overline{\mathcal{Z}}, \overline{\mathcal{K}}, \overline{\mathcal{A}})$ be oriented graphs. $\overline{\mathcal{G}}$ is a subgraph of $\mathcal{G}$ if $\overline{\mathcal{Z}} \subseteq \mathcal{Z}, \overline{\mathcal{K}} \subseteq \mathcal{K}$, and $\overline{\mathcal{A}}=\mathcal{A} \mid \overline{\mathcal{Z}}$. If $\mathcal{Z}^{\prime} \subseteq \mathcal{Z}, \mathcal{A}^{\prime}=\mathcal{A} \mid \mathcal{Z}^{\prime}$ and $\mathcal{K}^{\prime}=\{v \mid \exists b \in \mathcal{Z} v$ initial or terminal node of $b$ \} then $\mathcal{G}_{\mathcal{Z}^{\prime}}:=\left(\mathcal{Z}^{\prime}, \mathcal{K}^{\prime}, \mathcal{A}^{\prime}\right)$ is denoted as the subgraph of $\mathcal{G}$ generated by $\mathcal{Z}^{\prime}$.

As in Reibiger (1997); Reibiger et al. (1999); Reibiger (2003a,b) we define a network $\mathcal{N}$ as an ordered pair $\mathcal{N}=(\mathcal{C}, \mathcal{V})$ of a skeleton and a constitutive relation. The skeleton $\mathcal{C}$ is an ordered pair $\mathcal{C}=\left(\mathcal{G}_{\mathrm{v}}, \mathcal{G}_{\mathrm{c}}\right)$ of two oriented graphs with the same branch and node set differing at most in their orientations. $\mathcal{G}_{\mathrm{v}}$ and $\mathcal{G}_{\mathrm{c}}$ are denoted as the voltage or current graph of $\mathcal{N}$, respectively. The constitutive relation $\mathcal{V}$ is a restriction compatible nonvoid subset $\mathcal{V}$ of the universal signal set on the branch set of $\mathcal{G}_{\mathrm{v}}$ and $\mathcal{G}_{\mathrm{c}}$. For simplicity this signal set is denoted as the universal signal set of $\mathcal{N}$. Analogously, the branch and node sets of the graphs $\mathcal{G}_{\mathrm{v}}$ and $\mathcal{G}_{\mathrm{c}}$ are denoted as those of $\mathcal{N}$.

The skeleton describes the topological structure of a network. The constitutive relation characterizes the physical properties assigned to the branch set of a network. The constitutive relation is a set of ordered pairs of time functions and it is therefore a binary relation in the sense of set theory.

In the standard case the constitutive relation of a network is properly restriction compatible. The treatment of linear timeinvariant networks by means of Laplace transform, where only time functions defined on the intervall $[0,+\infty]$ are admitted, delivers typical examples of networks with trivially restriction compatible constitutive relations.

Let $\mathcal{N}$ be a network with branch set $\mathcal{Z}$. The elements of the universal signal set of $\mathcal{N}$ are denoted as signals. If $(u, i)$ is a signal then $u$ is called the corresponding voltage on the branch set of $\mathcal{N}$ and $i$ is called the corresponding current in the branch set of $\mathcal{N}$. For each signal $(u, i)$ and each $b \in \mathcal{Z}$, $\mathcal{Z}^{\prime} \subset \mathcal{Z}\left(\mathcal{Z}^{\prime} \neq \emptyset\right)$ the time functions $u_{b}, i_{b}, u_{\mathcal{Z}^{\prime}}$, and $i_{\mathcal{Z}^{\prime}}$ are denoted as the corresponding branch voltage, branch current, partial voltage on $\mathcal{Z}^{\prime}$, and partial current in $\mathcal{Z}^{\prime}$, respectively.

The orientations of the branches of the voltage and the current graph determine the reference directions for branch voltages and branch currents, respectively. The constitutive relation and both of the Kirchhoff's laws have reference to these orientations.
For the computer-aided analysis of networks it is indispensable to separate off the constitutive relation of a network from the corresponding universal signal set, or an appropriate subset of this set, by means of a constitutive equation. In analogy to Willems (1991); Poldermann and Willems (1998) the notion of a constitutive equation can be introduced as follows.

Let $\mathcal{N}=:(\mathcal{C}, \mathcal{V})$ be a network with universal signal set $\mathcal{S}$. If there exist a subset $\mathcal{S}^{\prime} \subseteq \mathcal{S}$ with $\mathcal{V} \subseteq \mathcal{S}^{\prime}$, an equating set $\mathcal{E}$, and two mappings $f, g: \mathcal{S}^{\prime} \rightarrow \mathcal{E}$ such that the relationship

$$
\mathcal{V}=\left\{(u, i) \in \mathcal{S}^{\prime} \mid f(u, i)=g(u, i)\right\}
$$

holds, then the equation $f(u, i)=g(u, i)$ is denoted as a constitutive equation of $\mathcal{N}$. The right hand side of Eq. (1) is denoted as a representation of the constitutive relation of $\mathcal{N}$ by means of a constitutive equation.

Let $\mathcal{N}$ be a network with universal signal set $\mathcal{S}$ and constitutive relation $\mathcal{V}$. Because the universal signal set contains a lot of very complicated signals, e.g. signals with nowhere differentiable voltages and currents, etc., it is useful to introduce in addition to the universal signal set some more specific signal sets. Of course, such a signal set $\mathcal{S}^{\prime}$ of $\mathcal{N}$ must be a subset of its universal signal set and it must contain its constitutive relation, $\mathcal{V} \subseteq \mathcal{S}^{\prime} \subset \mathcal{S}$. An important special case of such a signal set is a signal set with a prescribed type of branch signals.

Let $R: \mathcal{I} \rightarrow \mathcal{U}$ be a linear bijection then a set $\mathcal{S}_{*} \subseteq \bigcup_{T \in \operatorname{Int} \mathcal{T}}$ $\mathcal{U}^{T} \times \mathcal{I}^{T}$ is denoted as a branch signal type if it fulfills the conditions:

(i) $\mathcal{S}_{*}=\left\{(u, i) \mid u \in \mathcal{S}_{* \mathrm{v}} \wedge i \in \mathcal{S}_{* \mathrm{c}} \wedge \operatorname{dom} u=\operatorname{dom} i\right\}, \quad$ where $\mathcal{S}_{* \mathrm{v}}:=\left\{u \mid \exists_{i}(u, i) \in \mathcal{S}_{*}\right\}, \mathcal{S}_{* \mathrm{c}}:=\left\{i \mid \exists_{u}(u, i) \in \mathcal{S}_{*}\right\}$.

(ii) $\mathcal{S}_{*}=\left\{\left(R \circ i, R^{-1} \circ u\right) \mid(u, i) \in \mathcal{S}_{*}\right\}$,

(iii) $\mathcal{T} \in \operatorname{sd} \mathcal{S}_{*}$, where $\operatorname{sd} \mathcal{S}_{*}:=\left\{T \mid \exists_{u, i}(u, i) \in \mathcal{S}_{*} \wedge T=\operatorname{dom} u\right\}$.

(iv) $\forall_{(u, i) \in \mathcal{S}_{*}} \forall_{T \in \operatorname{Int}} \mathcal{T} T \subseteq \operatorname{dom} u \Rightarrow(u|T, i| T) \in \mathcal{S}_{*}$.

Typical examples of branch signal types are sets of ordered pairs of continously differentiable, piecewise continously differentiable functions, locally Riemann-integrable functions, etc.

Let $\mathcal{Z}$ be a finite set, $\mathcal{S}$ the universal signal set on $\mathcal{Z}$, and $\mathcal{S}_{*}$ a branch signal type. A set $\mathcal{S}^{\prime} \subseteq \mathcal{S}$ is called the $\mathcal{S}_{*}$-signal set on $\mathcal{Z}$ if $\mathcal{S}^{\prime}=\left\{(u, i) \in \mathcal{S} \mid \forall_{b \in \mathcal{Z}}\left(u_{b}, i_{b}\right) \in \mathcal{S}_{*}\right\}$.

Let $\mathcal{N}$ be a network whose constitutive relation is represented by means of an equating set $\mathcal{E}$ and a constitutive equation $f(u, i)=g(u, i)$ as a subset of some signal set $\mathcal{S}^{\prime}$. This constitutive equation is denoted as a constitutive equation in Belevitch form if the following additional conditions are fulfilled:

(i) The equating set $\mathcal{E}$ is a subset of $\bigcup_{T \in \operatorname{Int}} \mathcal{T}\left(\mathbb{R}^{m}\right)^{T}$, where $m$ is some natural number. 
(ii) The function $g$ is defined for all $(u, i) \in \mathcal{S}^{\prime}$ by $g(u, i):=0_{\operatorname{dom} u}$, where $0_{\operatorname{dom} u}$ denotes the zero function of $\left(\mathbb{R}^{m}\right)^{\operatorname{dom} u}$.

The resulting constitutive equation $f(u, i)=0_{\operatorname{dom} u}$ is usually simply written as $f(u, i)=0$.

In the standard case, for nondegenerated networks, the value of $m$ is equal to the number of branches of the network.

Constitutive equations in resistance, conductance, hybrid, or chain form are special cases of constitutive equations in Belevitch form.

The set $\mathcal{H}$ of all signals $(u, i)$ of a network where $u$ and $i$ fulfills Kirchhoff's voltage or current law, respectively, is denoted as the Kirchhoff part of its universal signal set. The intersection $\mathcal{L}:=\mathcal{H} \cap \mathcal{V}$ of Kirchhoff part and constitutive relation is denoted as the behavior or the solution set of that network.

Obviously, both Kirchhoff's laws lead for each network $\mathcal{N}$ to a finite system of homogeneous linear algebraic equations. Therefore, if the constitutive relation of $\mathcal{N}$ is represented by means of some system of constitutive equations, the problem of the determination of the solution set of $\mathcal{N}$ can be reduced to the determination of the solution set of the resulting system of equations. Such a resulting system of equations is denoted as a system of behavioral equations of $\mathcal{N}$.

Traditionally, matrix calculus is used for the formulation of constitutive and behavioral equations of networks. Using a numbering of the branches of the network under consideration it is always possible to transfer the representation of the elements of the universal signal set of a network into the language of matrix calculus. Let $\mathcal{Z}$ be the branch set of this network, $z:=|\mathcal{Z}|$, and $\zeta:\{1, \ldots, z\} \rightarrow \mathcal{Z}$ a bijection. The definitions $\boldsymbol{u}^{\zeta}:=\left(u_{\zeta(1)}, \ldots, u_{\zeta(z)}\right)^{\top}$ and $\boldsymbol{i}^{\zeta}:=\left(i_{\zeta(1)}, \ldots, i_{\zeta(z)}\right)^{\top}$ assign to each signal $(u, i)$ of the universal signal set on $\mathcal{Z}$ its matrix representation $\left(\boldsymbol{u}^{\zeta}, \boldsymbol{i}^{\zeta}\right)$ with respect to $\zeta$.

Above we have introduced the notion of a projection of a subset of the universal signal set of a network generated by a subset of the branch set of this network. The following definition makes an interesting use of this concept.

Let $\mathcal{N}$ and $\tilde{\mathcal{N}}$ be networks with branch sets $\mathcal{Z}$ and $\tilde{\mathcal{Z}}$, respectively, and solution sets $\mathcal{L}$ and $\tilde{\mathcal{L}}$, respectively, where $\mathcal{Z} \subset \tilde{\mathcal{Z}}$. We say that $\tilde{\mathcal{N}}$ generates the solution set of $\mathcal{N}$ if $\mathcal{L}=\tilde{\mathcal{L}}_{\mathcal{Z}}$.

A multipole is a network with a distingushed subset of its node set whose elements are used as accessible terminals for the interconnection with other networks.

A multiport is a multipole whose terminal set is partitioned into terminal pairs.

Obviously, each $n$-port can be identified with a $2 n$-pole.

A network with $n$ nodes is called an elementary n-pole if its graphs are forests whose branches connect these nodes. A network with $2 n$ nodes and $n$ branches is called an elementary n-port if its graphs are forests with one-branch components only.
On this basis it is possible to introduce special kinds of networks such as independent voltage and current sources, open and short circuits, nullators and norators, resistors, controlled sources, gyrators, inductors, coupled inductors, capacitors, etc.

Elementary $n$-poles deliver in some sense minimal models of technical devices with $n$ terminals (Reibiger (2003a); Koenig et al. (1967), too). But it should be noted that in the general case it is only possible to construct a minimal model for some real technical device if networks with sufficiently general constitutive relations are admitted. Otherwise one has to try to synthesize an approximate realization by means of an interconnection of networks with more restricted constitutive relations.

Let $\left(\mathcal{N}^{l}\right)_{l \in L}$ be a family of networks $\mathcal{N}^{l}=\left(\mathcal{C}^{l}, \mathcal{V}^{l}\right)$ with branch sets $\mathcal{Z}^{l}$ and node sets $\mathcal{K}^{l}$. This family of networks is denoted as interconnectable if $\forall_{k, l \in L, k \neq l}\left(\mathcal{Z}^{k} \cap \mathcal{Z}^{l}=\emptyset \wedge \mathcal{K}^{k} \cap \mathcal{Z}^{l}=\emptyset \wedge \mathrm{sd} \mathcal{V}^{k}=\mathrm{sd} \mathcal{V}^{l}\right)$.

An interconnection of such a family of networks can be determined with the help of an equivalence relation $\simeq_{\text {equ }}$ on the union $\overline{\mathcal{K}}:=\bigcup_{l \in L} \mathcal{K}^{l}$ of their node sets. Its skeleton is then defined by an identifiction of the elements of each of the equivalence classes $\mathcal{K}^{\prime} \in \overline{\mathcal{K}} / \simeq_{\text {equ }}$ and its constitutive relation is equal to $\left\{(u, i) \in \mathcal{S} \mid \forall_{l \in L}\left(u_{\mathcal{Z}^{l}}, i_{\mathcal{Z}^{l}}\right) \in \mathcal{V}^{l}\right\}$ where $\mathcal{S}$ denotes the universal signal sets on $\bigcup_{l \in L} \mathcal{Z}^{l}$. For details see Reibiger (2003b).

Sometimes it is appropriate to use a formal interconnection of a family of interconnectable networks. It is defined by means of the identical relation on the union of their node sets.

Obviously, each network can be represented both as an interconnection of elementary multipols and as an interconnection of elementary multiports, too.

Elementary multipoles and multiports are usually denoted as network elements in the literature. But in our formalization of network theory they are itself networks (obviously, networks with very simple skeletons) and they can be subnetworks of other, larger networks. Therefore we avoid to denote them as elements.

The notion of the terminal behavior of a network is of central importance as well for the application of network theory as for the further development of this theory, too. Indeed, a lot of theorems of network theory deliver propositions about the terminal behavior of some networks.

Let $\mathcal{N}$ be a given network with a prescribed subset of its node set whose elements are denoted as terminals. The terminal behavior of $\mathcal{N}$ has to characterize the behavior of this network with respect to all interconnections of its terminals with arbitrary admissible external networks $\tilde{\mathcal{N}}$ in such a manner that after an exchange of the given network $\mathcal{N}$ by another network $\hat{\mathcal{N}}$ with the same terminal behavior this exchange can not be observed in all the external networks $\tilde{\mathcal{N}}$ of these interconnections. 
For an introduction let us first assume that $\mathcal{N}$ is a network with at least two nodes where exactly two of its nodes are distingushed as terminals at which $\mathcal{N}$ can be interconnected with external networks $\tilde{\mathcal{N}}$. The terminal behavior of $\mathcal{N}$ can then be defined as the set of all ordered pairs of terminal pair voltages and terminal currents corresponding to the solution sets of all interconnections of the given network $\mathcal{N}$ with arbitrary external networks $\tilde{\mathcal{N}}$. Obviously, each such ordered pair of terminal pair voltages and terminal currents is also compatible with the interconnection of $\mathcal{N}$ with a norator. Because this norator is an element of the set of all admissible interconnections of $\mathcal{N}$ with external networks, it suffices to consider for the determination of the terminal behavior of $\mathcal{N}$ merely its interconnection with only one norator. The terminal behavior of $\mathcal{N}$ is then essentially equal to the projection of the solution set of this interconnection generated by the branch set of the external norator.

By means of this idea we have given in Reibiger (1997) a geometrical proof of the Thévenin-Norton theorem.

These considerations can be immediately generalized to the case of the determination of the terminal behavior of networks which are connected to external networks at more than two terminals. As it is shown in Reibiger $(1985,1986$, 2003a); Haase (1983) it suffices in this case to analyse the interconnection of a given network with a tree (and in more general cases with a forest) of norators connecting the terminals of the given network. These ideas are also applied in Clauß et al. (1995); Enge-Rosenblatt et al. (2007).

\section{Central results}

In this section we consider the relationship between four networks denoted as $\mathcal{N}, \mathcal{N}^{\text {aug }}, \overline{\mathcal{N}}$, and $\overline{\mathcal{N}}^{\text {aug }}$.

For this purpose we assume that there are given a branch signal type $\mathcal{S}_{*}$ and four finite pairwise disjoint nonvoid sets $\mathcal{Z}_{1}, \mathcal{Z}_{2}, \mathcal{Z}_{3}$, and $\mathcal{K}$ with $\left|\mathcal{Z}_{2}\right|=\left|\mathcal{Z}_{3}\right|$ and $|\mathcal{K}|=2\left|\mathcal{Z}_{1} \cup \mathcal{Z}_{2}\right|$. The $\mathcal{S}_{*}$-signal sets on $\mathcal{Z}:=\mathcal{Z}_{1} \cup \mathcal{Z}_{2}$ and $\mathcal{Z}^{\text {aug }}:=\mathcal{Z}_{1} \cup \mathcal{Z}_{2} \cup \mathcal{Z}_{3}$ are denoted with $\mathcal{S}$ and $\mathcal{S}$ aug, respectively.

The network $\mathcal{N}$ is defined as an elementary multiport with branch set $\mathcal{Z}$, node set $\mathcal{K}$, and a constitutive relation which can be represented by means of the $\mathcal{S}_{*}$-signal set $\mathcal{S}$ as the set of all signals $(u, i) \in \mathcal{S}$ obeying the semi-implicit constitutive equations

$$
\begin{aligned}
i_{\mathcal{Z}_{1}} & =g\left(u_{\mathcal{Z}_{1}}, u_{\mathcal{Z}_{2}}, i_{\mathcal{Z}_{2}}\right), \\
0 & =h\left(u_{\mathcal{Z}_{1}}, u_{\mathcal{Z}_{2}}, i_{\mathcal{Z}_{2}}\right),
\end{aligned}
$$

where

$$
g:\left\{\left(u_{\mathcal{Z}_{1}}, u_{\mathcal{Z}_{2}}, i_{\mathcal{Z}_{2}}\right) \mid(u, i) \in \mathcal{S}\right\} \rightarrow\left\{i_{\mathcal{Z}_{1}} \mid(u, i) \in \mathcal{S}\right\}
$$

and

$$
h:\left\{\left(u_{\mathcal{Z}_{1}}, u_{\mathcal{Z}_{2}}, i_{\mathcal{Z}_{2}}\right) \mid(u, i) \in \mathcal{S}\right\} \rightarrow\left\{i_{\mathcal{Z}_{3}} \mid(u, i) \in \mathcal{S}^{\text {aug }}\right\} .
$$

The network $\mathcal{N}^{\text {aug }}$ is defined as a meshless multipole with branch set $\mathcal{Z}^{\text {aug }}$ and terminal set $\mathcal{K}$ satisfying the following conditions: The voltage and current graph of $\mathcal{N}^{\text {aug }}$ consist of at least $|\mathcal{Z}|$ components and and each of these components includes at most one branch of $\mathcal{Z}$. The subgraphs of these graphs generated by $\mathcal{Z}$ are equal to the corresponding graphs of $\mathcal{N}$. Its constitutive relation can be represented by means of the $\mathcal{S}_{*}$-signal set $\mathcal{S}^{\text {aug }}$ together with a linear bijection $G: \mathcal{U}^{\mathcal{Z}_{3}} \rightarrow \mathcal{I}^{\mathcal{Z}_{2}}$ as the set of all signals $(u, i) \in \mathcal{S}^{\text {aug }}$ obeying the following constitutive equations in conductance form

$$
\begin{aligned}
& i_{\mathcal{Z}_{1}}=g\left(u_{\mathcal{Z}_{1}}, u_{\mathcal{Z}_{2}}, G \circ u_{\mathcal{Z}_{3}}\right), \\
& i_{\mathcal{Z}_{2}}=G \circ u_{\mathcal{Z}_{3}}, \\
& i_{\mathcal{Z}_{3}}=h\left(u_{\mathcal{Z}_{1}}, u_{\mathcal{Z}_{2}}, G \circ u_{\mathcal{Z}_{3}}\right) .
\end{aligned}
$$

Theorem 1: The networks $\mathcal{N}$ and $\mathcal{N}^{\text {aug }}$ have the same terminal behavior with respect to $\mathcal{K}$.

PROOF: For a proof of this theorem we connect $\mathcal{N}$ and $\mathcal{N}^{\text {aug }}$ with a norator tree consisting of $|\mathcal{K}|-1$ branches such that in the resulting network exactly one branch of this norator network is parallel to a branch of $\mathcal{Z}$ and all these parallel connections are pairwise connected by means of one of the remaining norators.

Then both of these interconnections are analyzed. It is easy to see that the projections of the solution sets of these interconnections generated by the branch set of the norator-tree are equal. That means, $\mathcal{N}$ and $\mathcal{N}^{\text {aug }}$ have the same terminal behavior.

Remark 1: Theorem 1 and both of the subsequent theorems, too, can be generalized to the case $\mathcal{Z}_{1}=\emptyset$. Then the Eqs. (2) and (4) are to delete and in the remaining equations the term $u_{\mathcal{Z}_{1}}$ must be omitted.

Remark 2: Let $\left(\mathcal{N}^{l}\right)_{l \in L}$ be a family of interconnectable elementary multiports whose constitutive relations satisfy the assumptions of Theorem 1. Then the formal interconnection of these multiports is an elementary multiport, too, fulfilling these assumptions likewise.

Let $\simeq_{\text {equ }}$ denote an equivalence relation defined on the terminal set of $\mathcal{N}$ and $\mathcal{N}^{\text {aug }}$. The networks $\overline{\mathcal{N}}$ and $\overline{\mathcal{N}}^{\text {aug }}$ are then defined by an identification of the elements of each of the equivalence classes $\mathcal{K}^{\prime} \in \mathcal{K} / \simeq_{\text {equ }}$.

Theorem 2: The network $\overline{\mathcal{N}}^{\text {aug }}$ generates the solution set of $\overline{\mathcal{N}}$.

Our proof of this theorem is based on the following lemma. Its proof follows directly from the fact that the branches of $\mathcal{Z}_{3}$ are not included in any mesh of $\overline{\mathcal{N}}^{\text {aug }}$. For the formulation of this lemma we need some additional notations. 
We set $z_{1}:=\left|\mathcal{Z}_{1}\right|, \quad z_{2}:=\left|\mathcal{Z}_{2}\right|, \quad z_{3}:=\left|\mathcal{Z}_{3}\right|, \quad z:=|\mathcal{Z}|=z_{1}+z_{2}$. Nullity and rank of the graphs of $\overline{\mathcal{N}}$ are denoted by $n$ and $r$, respectively. $M$ denotes a reduced mesh-branch incidence matrix of the voltage graph of $\overline{\mathcal{N}}$ and $K$ a reduced node-branch incidence matrix of the current graph of $\overline{\mathcal{N}}$. By definition, $M$ is an $n \times z$ and $K$ an $r \times z$ matrix. The assignment of the columns of these matrices to the branch set of $\overline{\mathcal{N}}$ is given by a bijective numbering $\zeta:\{1, \ldots, z\} \rightarrow \mathcal{Z}$ satisfying $\zeta\left(\left\{1, \ldots, z_{1}\right\}\right)=\mathcal{Z}_{1}$ and $\zeta\left(\left\{z_{1}+1, \ldots, z\right\}\right)=\mathcal{Z}_{2}$.

Lemma: Suppose $\left(\begin{array}{ll}M_{1} & M_{2}\end{array}\right)$ denote the natural partition of the matrix $M$ corresponding to the partition $\left(\mathcal{Z}_{1}, \mathcal{Z}_{2}\right)$ of $\mathcal{Z}$, then there exists an $n \times z_{3}$ zero matrix 0 such that

$$
\left(\begin{array}{lll}
M_{1} & M_{2} & 0
\end{array}\right)
$$

is a reduced mesh-branch incidence matrix of the voltage graph of $\overline{\mathcal{N}}^{\text {aug }}$.

Analogously, if $\left(\begin{array}{ll}K_{1} & K_{2}\end{array}\right)$ denotes the natural partion of $K$ corresponding to the partition $\left(\mathcal{Z}_{1}, \mathcal{Z}_{2}\right)$ of $\mathcal{Z}$, then there exist appropriate zero matrices and a regular $z_{3} \times z_{3}$ matrix $K_{3}$ such that

$$
\left(\begin{array}{ccc}
K_{1} & K_{2} & 0 \\
0 & 0 & K_{3}
\end{array}\right)
$$

is a conformably partitioned reduced cut-branch incidence matrix of the current graph of $\mathcal{N}^{\text {aug }}$.

Proof of THEOREM 2: For simplicity we identify in the following the partial voltages and currents of each signal $(u, i)$ of the universal signal set of $\mathcal{N}^{\text {aug }}$ corresponding to the branch sets $\mathcal{Z}_{1}, \mathcal{Z}_{2}$, and $\mathcal{Z}_{3}$ with column matrices of length $z_{1}, z_{2}$, and $z_{3}$, resp.

Obviously, the constitutive Eqs. (4), (5), and (6) deliver together with

$$
\left(\begin{array}{lll}
M_{1} & M_{2} & 0
\end{array}\right)\left(\begin{array}{l}
u_{\mathcal{Z}_{1}} \\
u_{\mathcal{Z}_{2}} \\
u_{\mathcal{Z}_{3}}
\end{array}\right)=0
$$

and

$$
\left(\begin{array}{ccc}
K_{1} & K_{2} & 0 \\
0 & 0 & K_{3}
\end{array}\right)\left(\begin{array}{l}
i_{\mathcal{Z}_{1}} \\
i_{\mathcal{Z}_{2}} \\
i_{\mathcal{Z}_{3}}
\end{array}\right)=0
$$

a system of behavioral equations for $\overline{\mathcal{N}}^{\text {aug }}$. Let $(u, i)$ be a solution of $\overline{\mathcal{N}}^{\text {aug }}$, i.e., a solution of the system of the Eqs. (4) to (8). Then the Eqs. (7) and (8) imply

$$
\left(\begin{array}{ll}
M_{1} & M_{2}
\end{array}\right)\left(\begin{array}{l}
u_{\mathcal{Z}_{1}} \\
u_{\mathcal{Z}_{2}}
\end{array}\right)=0
$$

and

$$
\left(\begin{array}{ll}
K_{1} & K_{2}
\end{array}\right)\left(\begin{array}{l}
i_{\mathcal{Z}_{1}} \\
i_{\mathcal{Z}_{2}}
\end{array}\right)=0
$$

Since $K_{3}$ is regular, it follows from Eq. (8) that

$$
i_{\mathcal{Z}_{3}}=0
$$

and therefore, together with Eqs. (5) and (6), that $u_{\mathcal{Z}_{1}}, u_{\mathcal{Z}_{2}}$, $i_{\mathcal{Z}_{1}}$, and $i_{\mathcal{Z}_{2}}$ satisfy the Eqs. (2) and (3). But the Eqs. (2), (3), (9) and (10) are a system of behavioral equations of for $\overline{\mathcal{N}}$. That means, $\left(u_{\mathcal{Z}}, i_{\mathcal{Z}}\right)$ is a solution of $\overline{\mathcal{N}}$.

Let $\mathcal{K}_{\text {dat. }}$ be an arbitrary chosen equivalence class of the quotient set $\mathcal{K} / \simeq_{\text {equ }}$ and let $n_{\text {dat. }}$ be that node of $\overline{\mathcal{N}}$ and $\overline{\mathcal{N}}^{\text {aug }}$ corresponding to this equivalence class.

Theorem 3: If the branches of $\mathcal{Z}_{3}$ generate starlike subtrees of the voltage and current graph of $\overline{\mathcal{N}}^{\text {aug }}$ with $n_{\text {dat. }}$ as center node, then the system of nodal voltage equations for $\overline{\mathcal{N}}^{\text {aug }}$ with respect to $n_{\text {dat. }}$ as datum node is equivalent to a system of modified nodal voltages for $\overline{\mathcal{N}}$.

PROOF: Our proof consists of two parts.

Part 1: Analysis of $\overline{\mathcal{N}}$ by means of a system of $u-i-u^{\varnothing}$ equations:

$$
\begin{gathered}
\left(\begin{array}{l}
u_{\mathcal{Z}_{1}} \\
u_{\mathcal{Z}_{2}}
\end{array}\right)=\left(\begin{array}{l}
K_{1}^{\top} \\
K_{2}^{\dagger}
\end{array}\right) u^{\emptyset}, \\
i_{\mathcal{Z}_{1}}=g\left(u_{\mathcal{Z}_{1}}, u_{\mathcal{Z}_{2}}, i_{\mathcal{Z}_{2}}\right), \\
0=h\left(u_{\mathcal{Z}_{1}}, u_{\mathcal{Z}_{2}}, i_{\mathcal{Z}_{2}}\right), \\
\left(\begin{array}{ll}
K_{1} & K_{2}
\end{array}\right)\left(\begin{array}{c}
i_{\mathcal{Z}_{1}} \\
i_{\mathcal{Z}_{2}}
\end{array}\right)=0 .
\end{gathered}
$$

Elimination of $u_{\mathcal{Z}_{1}}, u_{\mathcal{Z}_{2}}$, and $i_{\mathcal{Z}_{1}}$ delivers a system of modified nodal voltage equations for $\overline{\mathcal{N}}$ :

$$
\begin{aligned}
K_{1} g\left(K_{1}^{\top} u^{\emptyset}, K_{2}^{\top} u^{\emptyset}, i_{\mathcal{Z}_{2}}\right)+K_{2} i_{\mathcal{Z}_{2}} & =0, \\
h\left(K_{1}^{\top} u^{\varnothing}, K_{2}^{\top} u^{\emptyset}, i_{\mathcal{Z}_{2}}\right) & =0 .
\end{aligned}
$$

Part 2: Analysis of $\overline{\mathcal{N}}^{\text {aug }}$ by means of nodal voltage equations:

Under the assumptions of Theorem 3 the branches of $\mathcal{Z}_{3}$ generate starlike subtrees with the datum node as center node. Therefore the submatrix $K_{3}$ of the node-branch incidence matrix of $\overline{\mathcal{N}}^{\text {aug }}$ is equal to a $z_{3} \times z_{3}$ unit matrix $E_{3}$ and it follows

$$
\left(\begin{array}{l}
u_{\mathcal{Z}_{1}} \\
u_{\mathcal{Z}_{2}} \\
u_{\mathcal{Z}_{3}}
\end{array}\right)=\left(\begin{array}{cc}
K_{1}^{\top} & 0 \\
K_{2}^{\top} & 0 \\
0 & E_{3}
\end{array}\right)\left(\begin{array}{l}
u_{1}^{\varnothing} \\
u_{2}^{\Phi}
\end{array}\right),
$$

$$
\begin{aligned}
& i_{\mathcal{Z}_{1}}=g\left(u_{\mathcal{Z}_{1}}, u_{\mathcal{Z}_{2}}, i_{\mathcal{Z}_{2}}\right), \\
& i_{\mathcal{Z}_{2}}=G \circ u_{\mathcal{Z}_{3}}, \\
& i_{\mathcal{Z}_{3}}=h\left(u_{\mathcal{Z}_{1}}, u_{\mathcal{Z}_{2}}, i_{\mathcal{Z}_{2}}\right),
\end{aligned}
$$

$$
\left(\begin{array}{ccc}
K_{1} & K_{2} & 0 \\
0 & 0 & E_{3}
\end{array}\right)\left(\begin{array}{l}
i_{\mathcal{Z}_{1}} \\
i_{\mathcal{Z}_{2}} \\
i_{\mathcal{Z}_{3}}
\end{array}\right)=0
$$


Obviously, $i_{\mathcal{Z}_{3}}=0$. Elimination of $i_{\mathcal{Z}_{2}}$ and $u_{\mathcal{Z}_{3}}$ yields

$$
\begin{aligned}
K_{1} g\left(K_{1}^{\top} u_{1}^{\emptyset}, K_{2}^{\top} u_{2}^{\emptyset}, G \circ u_{2}^{\varnothing}\right)+K_{2} G \circ u_{2}^{\varnothing} & =0, \\
h\left(K_{1}^{\top} u_{1}^{\emptyset}, K_{2}^{\top} u_{2}^{\Phi}, G \circ u_{2}^{\Phi}\right) & =0 .
\end{aligned}
$$

It is easy to see that the assignment $\left(u^{\varnothing}, i_{\mathcal{Z}_{2}}\right) \mapsto\left(u_{1}^{\varnothing}, u_{2}^{\phi}\right)$ defined by $u_{1}^{\varnothing}:=u^{\varnothing}$ and $u_{2}^{\emptyset}:=G^{-1} \circ i_{\mathcal{Z}_{2}}$ delivers a bijection between the solution sets of the equation systems Eqs. (11) and (12), and Eqs. (13) and (14).

The modified nodal voltage Eqs. (11) and (12) are set up here under the assumption that the constitutive equations of $\overline{\mathcal{N}}$ are the semi-implicit Eqs. (2) and (3). However, in the literature it is for this purpose usually assumed that the constitutive equations are given in hybrid form. Observe, the constitutive equations $u_{1}=0, i_{1}=0$ of a nullor are not in hybrid form.

\section{Examples}

$\left(1^{\circ}\right)$ Nullor: A nullor is an elementary two-port. If $\mathcal{Z}:=\{1,2\}$ denotes its branch set, then its constitutive relation can be characterized by the equations

$$
u_{1}=0, \quad i_{1}=0
$$

or equivalently

$$
i_{1}=0, \quad 0=G \circ u_{1},
$$

where $G: \mathcal{U} \rightarrow \mathcal{I}$ linear bijection. Therefore the associated network $\mathcal{N}^{\text {aug }}$ can defined by

$$
\begin{aligned}
& \mathcal{Z}_{1}=\{1\}, \quad \mathcal{Z}_{2}=\{2\}, \quad \mathcal{Z}_{3}=\{3\}, \\
& i_{1}=0, \quad i_{2}=G \circ u_{3}, \quad i_{3}=G \circ u_{1} .
\end{aligned}
$$

$\left(2^{\circ}\right)$ Independent Voltage Source: An independent voltage source is an elementary one-port. If $\mathcal{Z}:=\{1\}$ denotes its branch set, then its constitutive relation can be together with a prescribed voltage $u_{1}^{\mathrm{e}} \in \mathcal{S}_{*}$ characterized by the equation

$$
u_{1}=u_{1}^{\mathrm{e}} \mid \operatorname{dom} u_{1},
$$

or equivalently

$$
0=G \circ\left(u_{1}-u_{1}^{\mathrm{e}} \mid \operatorname{dom} u_{1}\right),
$$

where $G: \mathcal{U} \rightarrow \mathcal{I}$ linear bijection. Therefore the associated network $\mathcal{N}^{\text {aug }}$ can be defined by

$$
\begin{gathered}
\mathcal{Z}_{1}=\emptyset, \quad \mathcal{Z}_{2}=\{1\}, \quad \mathcal{Z}_{3}=\{2\}, \\
i_{1}=G \circ u_{2}, \quad i_{2}=G \circ\left(u_{1}-u_{1}^{\mathrm{e}} \mid \operatorname{dom} u_{1}\right) .
\end{gathered}
$$

$\left(3^{\circ}\right)$ Current Controlled Voltage Source: A current controlled voltage source is an elementary two-port. If $\mathcal{Z}:=\{1,2\}$ denotes its branch set, then its constitutive relation can be characterized by the equations

$$
u_{1}=0, \quad u_{2}=R_{\mathrm{trf}} \circ i_{1}
$$

or equivalently

$$
0=G \circ u_{1}, \quad 0=G \circ u_{2}-G \circ R_{\mathrm{trf}} \circ i_{1},
$$

where $R_{\text {trf }}: \mathcal{I} \rightarrow \mathcal{U}$ is an arbitrary map and $G: \mathcal{U} \rightarrow \mathcal{I}$ is a linear bijection. Therefore the associated network $\mathcal{N}^{\text {aug }}$ can be defined by

$$
\begin{gathered}
\mathcal{Z}_{1}=\emptyset, \quad \mathcal{Z}_{2}=\{1,2\}, \quad \mathcal{Z}_{3}=\{3,4\}, \\
i_{1}=G \circ u_{3}, \quad i_{2}=G \circ u_{4}, \quad i_{3}=G \circ u_{1} \\
i_{4}=G \circ u_{2}-G \circ R_{\text {trf }} \circ i_{1} .
\end{gathered}
$$

If $R_{\mathrm{trf}}$ is a bijection, too, then it is possible to start from the equations

$$
i_{1}=R_{\text {trf }}^{-1} \circ u_{2}, \quad 0=G \circ u_{1} .
$$

The associated network $\mathcal{N}^{\text {aug }}$ can now be defined by

$$
\begin{gathered}
\mathcal{Z}_{1}=\{1\}, \quad \mathcal{Z}_{2}=\{2\}, \quad \mathcal{Z}_{3}=\{3\}, \\
i_{1}=R_{\text {trf }}^{-1} \circ u_{2}, \quad i_{2}=G \circ u_{3}, \quad i_{3}=G \circ u_{1} .
\end{gathered}
$$

\section{Concluding remarks}

On the basis of some recent results on the foundations of network theory we have presented an algorithm for the construction of a multipole having the same all-pole terminal behavior as an given elementary multiport whose constitutive relation is defined by means of a system of semi-implicit constitutive equations such that the constitutive relation of the modified multipole can be represented by means of constitutive equations in conductance form.

A row of other applications of the auxiliary branch method to circumvent restrictions of the input languages of circuit simulators can you find in Haase (1983); Clauß et al. (1995); Reibiger and Elst (1983).

\section{References}

Clauß, C., Haase, J., Kurth, G., and Schwarz, P.: Extended Admittance Description of Nonlinear n-Poles, AEU-Arch. Elektron. Ub., 49, 91-97, 1995.

Enge-Rosenblatt, O., Haase, J., and Clauß, C.: Important Characteristics of VHDL-AMS and Modelica with Respect to Model Exchange, EOOLT Workshop, Berlin, Juli 2007, 2007.

Engelhardt, R. and Heinz, M.: Behandlung idealer gesteuerter Quellen bei der Netzwerkanalyse mit Digitalrechnern, Nachrichtentech. Z., 23, 8-10, 1970.

Haase, J.: Verfahren zur Beschreibung und Berechnung des Klemmenverhaltens resistiver Netzwerke, Diss. TU Dresden, 1983.

Klein, W.: Der Übertrager als Zweipolnetz, AEU-Arch. Elektron. Ub., 12, 133-137, 1958.

Koenig, H. E., Tokad, Y., Kesavan, H. K., and Hedges, H. G.: Analysis of Discrete Physical Systems, McGraw-Hill, 1967.

Kremer, H.: Numerische Berechnung linearer Netzwerke und Systeme, Springer-Verlag, 1978. 
Poldermann, J. W. and Willems, J. C.: Introduction to Mathematical Systems Theory - A Behavioral Approach, Springer, 1998.

Reibiger, A.: On the Terminal Behaviour of Networks, Proc. ECCTD'85, Prague, 224-227, 1985.

Reibiger, A.: Über das Klemmenverhalten von Netzwerken, Wiss. Z. TU Dresden, 35, 165-173, 1986.

Reibiger, A.: Geometrical Proof of the Thévenin-Norton Theorem", Proc. ECCTD'97, Budapest, 1, 24-28, 1997.

Reibiger, A.: Terminal Behaviour of Networks, Multipoles and Multiports, Proc. 4th MATHMOD, Vienna, (CD), 2003a.

Reibiger, A.: Networks, Decomposition and Interconnection of Networks, Proc. ISTET'03, Warsaw, 245-248, 2003 b.

Reibiger, A. and Elst, G.: Network Theoretic Interpretation of Multibody Systems, Acta Polytechnica Práce ČVUT v Praze, 18, (III,4), Práce, 109-129, 1983.

Reibiger, A. and Loose, H.: Bond Graphs and Matroids (cf. also Proc. 3rd MATHMOD, Vienna, 2000), Math. Comput. Simulat., 53, 323-332, 2000.
Reibiger, A., Loose, H., and Nähring, T.: Multidimensional Networks, Proc. X. Int. Symp. Theor. Electrical Engineering, ISTET'99, Magdeburg, 293-298, 1999.

Reibiger, A., Mathis, W., Nähring, T., and Trajkovi'c, Lj.: Mathematical Foundations of the TC-Method for Computing Multiple DC-Operating Points, Int. J. Appl. Electrom., 17, 169-191, 2003.

Reinschke, K. and Schwarz, P.: Verfahren zur rechnergestützten Analyse linearer Netzwerke, Akademie-Verlag, 1976.

Reinschke, K. and Schwarz, P.: Computer-Aided Analysis of Linear Networks with Ideal Elements, Proc. SSCT 77, Kladno, 506511, 1977.

Thielmann, H.: Analysis of Linear Active $(n+1)-P o l e s$ by Simple Transformations of the Nodal Admittance Matrix, IEEE Trans. Circ. Theory CT-20, 575-577, 1973.

Willems, J. C.: Paradigms and Puzzles in the Theory of Dynamical Systems, IEEE T. Automat. Contr., AC-36, 259-294, 1991. 